\title{
Telomerase regulation by the Pif1 helicase: a length-dependent effect?
}

\author{
Sonia Stinus $^{1} \cdot$ Katrin Paeschke $^{1} \cdot$ Michael Chang $^{1}$ (I)
}

Received: 29 September 2017 / Revised: 13 October 2017 / Accepted: 15 October 2017 / Published online: 20 October 2017

(C) The Author(s) 2017. This article is an open access publication

\begin{abstract}
Dysfunctional telomere length regulation is detrimental to human health, and both activation and inhibition of telomerase have been proposed in potential therapies to treat human diseases. The Saccharomyces cerevisiae Pif1 protein is an evolutionarily conserved helicase that inhibits telomerase activity at DNA ends. Recent studies have indicated that Pif1 is specifically important for inhibiting telomerase at DNA ends with very little or no telomeric sequence and at long telomeres. At the former, Pif1 prevents the inappropriate addition of a telomere at DNA doublestrand breaks. For the latter, Pif1 has been shown to bind long telomeres to presumably promote the extension of the short ones. These observations leave the impression that Pif1 does not act at DNA ends with telomeric sequence of intermediate length. Here, we provide in vivo evidence that Pif1 inhibits telomerase activity at DNA ends regardless of telomere sequence length.
\end{abstract}

Keywords Pif $1 \cdot$ Telomeres $\cdot$ Telomerase $\cdot$ Telomere length homeostasis $\cdot$ Double-strand breaks

The ends of eukaryotic chromosomes are capped by telomeres, nucleoprotein complexes that protect chromosome ends from degradation, and telomere-telomere fusion (Ferreira et al. 2004). Telomeres can shorten due to incomplete

Communicated by M. Kupiec.

Michael Chang

m.chang@umcg.nl

1 European Research Institute for the Biology of Ageing, University Medical Center Groningen, University of Groningen, A. Deusinglaan 1, 9713 AV Groningen, The Netherlands replication or nuclease-dependent degradation. This shortening is counteracted by a specialized reverse transcriptase called telomerase (Greider and Blackburn 1985). The consequences of improper telomere length regulation are severe: several human diseases are linked to telomere shortening (reviewed in Blasco 2005; Armanios and Blackburn 2012), and upregulation of telomerase is a common feature in cancer cells (Kim et al. 1994).

Telomere length homeostasis maintenance is a tightly regulated process and has been best studied in the budding yeast Saccharomyces cerevisiae, whose telomeres consist of $300 \pm 75 \mathrm{bp}$ of $\mathrm{C}_{1-3} \mathrm{~A} / \mathrm{TG}_{1-3}$ repeats (Wellinger and Zakian 2012). Not all telomeres are elongated during each cell cycle and the probability of extension of a given telomere is inversely correlated to its length, with shorter telomeres preferentially elongated (Teixeira et al. 2004). In budding yeast, the double-stranded telomeric DNA-binding protein Rap1 forms a complex together with Rif1 and Rif2 to negatively regulate telomerase (Wotton and Shore 1997). According to the protein-counting model (Marcand et al. 1997), telomere-bound Rap1 increases proportionally with telomere length, preventing telomerase from extending the longer telomeres, thereby providing a mechanism to maintain telomere length homeostasis.

The evolutionarily conserved Pif1 helicase is also an important player in telomere length regulation. In vitro, Pif1 reduces the nucleotide addition processivity of telomerase, removes telomerase from telomere-like oligonucleotides (Boulé et al. 2005), and unwinds the telomere-telomerase complex ( $\mathrm{Li}$ et al. 2014). In vivo, mutation of PIF 1 causes telomere lengthening (Schulz and Zakian 1994) and increases telomere association of the telomerase subunits, Est1 and Est2 (Boulé et al. 2005; Phillips et al. 2015). On the other hand, Pif1 overexpression has been shown to cause telomere shortening (Zhou et al. 2000) and to reduce Est1 
and Est2 binding to chromosome ends (Boulé et al. 2005). Taken together, the evidence suggests that Pif1 regulates telomere length by unwinding the DNA-RNA hybrid formed by the telomeric DNA and telomerase RNA subunit.

Besides telomere length regulation, Pif1 function is also important at double-strand DNA breaks (DSBs). Gross chromosomal rearrangements (GCR) are known to be increased 230 -fold in pifl- $m 2$ cells, where only the nuclear function and not the mitochondrial function of Pif1 is affected (Schulz and Zakian 1994), and 1000-fold in pifl $1 \Delta$ cells (Myung et al. 2001). The increase of GCR events in pifl- $m 2$ cells is due to improper and deleterious addition of telomere sequence, and deletion of telomerase subunits rescues the GCR rate to wild-type levels (Myung et al. 2001). Pif1 is thought to prevent telomere sequence addition at DSBs in cooperation with Mec1-dependent inhibition of the single-stranded telomeric DNA-binding protein Cdc13, which is needed for the recruitment of telomerase (Nugent et al. 1996). In response to DNA damage, $\mathrm{Cdc13}$ and Pif1 are phosphorylated in a Mec1-dependent manner to preserve genome stability. Phosphorylation of $\mathrm{Cdc} 13$ inhibits its accumulation at DSBs, thus preventing telomerase recruitment to these sites, and phosphorylated Pif1 removes telomerase from DSBs (Makovets and Blackburn 2009; Zhang and Durocher 2010).

Remarkably, although Pif1 functions both at DSBs and at telomeres, it is differently regulated at these two DNA ends. Two phosphorylation mutants of Pif1 support this idea: the unphosphorylatable pif1-4A mutant (T763A/S765A/S766A/ S769A) is unable to inhibit telomere addition at DSBs, but it does not result in bulk telomere lengthening at chromosome ends, whereas the pifl-4D mutant, which mimics constitutive phosphorylation, restores Pif1 activity at DSBs but does not give rise to shorter telomeres. This observation implies that phosphorylation of Pif1 is important for inhibiting telomerase at DSBs and not at telomeres (Makovets and Blackburn 2009).

In a recent study, Pif 1 was reported to be found preferentially at long telomeres, and it was suggested that this serves to promote telomerase-dependent extension of short telomeres (Phillips et al. 2015). We have also recently published a study where the inducible HO cut system was used to create DNA ends adjacent to telomeric sequence of different length to monitor the ability of Pif1 to inhibit telomerase. We found that a 34-bp telomere tract is enough to render such an end insensitive to Pif1, resulting in a very high frequency of telomere addition. We defined this tract length as the DSB-telomere threshold, below which Pif1 actively supresses telomere addition (Strecker et al. 2017). This result suggests that Pif 1 activity becomes irrelevant at telomeres longer than $34 \mathrm{bp}$.

Together, the two above-mentioned studies give the impression that Pif1 activity is only relevant in two scenarios: (1) at DNA ends with no telomeric sequence or with telomeric sequence shorter than the DSB-telomere threshold length, where its telomerase inhibitory activity protects genome stability by preventing inappropriate addition of telomeric sequences at DSBs, and (2) at long telomeres, where Pif1 inhibits telomerase to facilitate the extension of short telomeres. However, our data show that the telomerase inhibitory activity of Pif1 takes place at all DNA ends, regardless of telomere length, and it is, therefore, telomere length independent.

We monitored telomerase-dependent telomere extension events in wild-type and pif $1-m 2$ cells using the iSTEX assay, which allows us to detect telomere extension events at individual telomeres at nucleotide resolution during a single cell cycle (Strecker et al. 2017). In wild-type cells, as previously reported, the frequency of telomere extension increases as telomere length decreases, and there is an increase in the extent of elongation at telomeres below $125 \mathrm{nt}$ (Teixeira et al. 2004). In pifl- $m 2$ cells, the extent of elongation at telomeres below $125 \mathrm{nt}$ is even greater (Fig. 1a, b). Yeast telomerase is generally non-processive in terms of repeat addition, except at telomeres less than $125 \mathrm{nt}$ in length, where an increase in repeat addition processivity leads to an increase in extension length (Chang et al. 2007). It is not known how this increased processivity is achieved, although it is known to be dependent on Tel1 (Chang et al. 2007), and we now propose that it is also inhibited by Pif1. The extent of elongation is not significantly different at telomeres greater than $125 \mathrm{nt}$ in pifl- $m 2$ cells, indicating that the lack of repeat addition processivity at such telomeres is not due to the presence of Pif1. Furthermore, we found an increase in the frequency of extension at telomeres of all length, but short telomeres were still preferentially extended (Fig. 2a, b). This implies that telomerase activity is inhibited by Pif 1 in a telomere length-independent fashion.

This finding is in agreement with a previous study showing that the frequency and extent of elongation of telomeres between 50 and $200 \mathrm{nt}$ in length was increased in pifl $\Delta$ cells (Phillips et al. 2015). The same study also reported increased Est 1 and Est 2 binding to an $\mathrm{HO}$-induced $\mathrm{TG}_{80}$ end, which mimics a short telomere, in pifl-m2 cells compared to the wild-type control. Intriguingly, Pif1 was also found to preferentially bind long telomeres (Phillips et al. 2015). If Pif1-mediated inhibition of telomerase is independent of telomere length, then why is Pif1 preferentially bound to long telomeres? Pif1 has several other functions in addition to telomerase inhibition. Pif 1 is also known to function in Okazaki fragment processing (Budd et al. 2006) and to promote replication of hard-to-replicate sites, such as telomeres (Paeschke et al. 2011). Thus, the interpretation of Pif1 binding to telomeres is complicated by the telomerase-independent functions of Pif 1 at telomeres. Longer telomeres would mean more DNA to be replicated, and may, therefore, require more Pif1. 


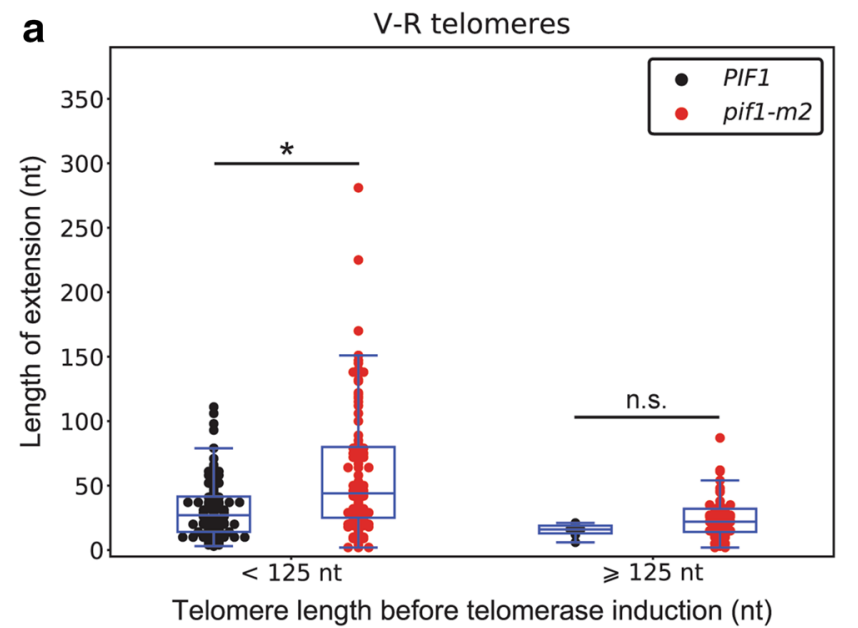

Fig. 1 Mutation of PIF1 increases telomere extension length at telomeres less than $125 \mathrm{nt}$ in length. Length of telomere extension for telomeres V-R (a) and VI-R (b), obtained from iSTEX analysis of wild-type and pif1-m2 cells (Strecker et al. 2017), is plotted accord-

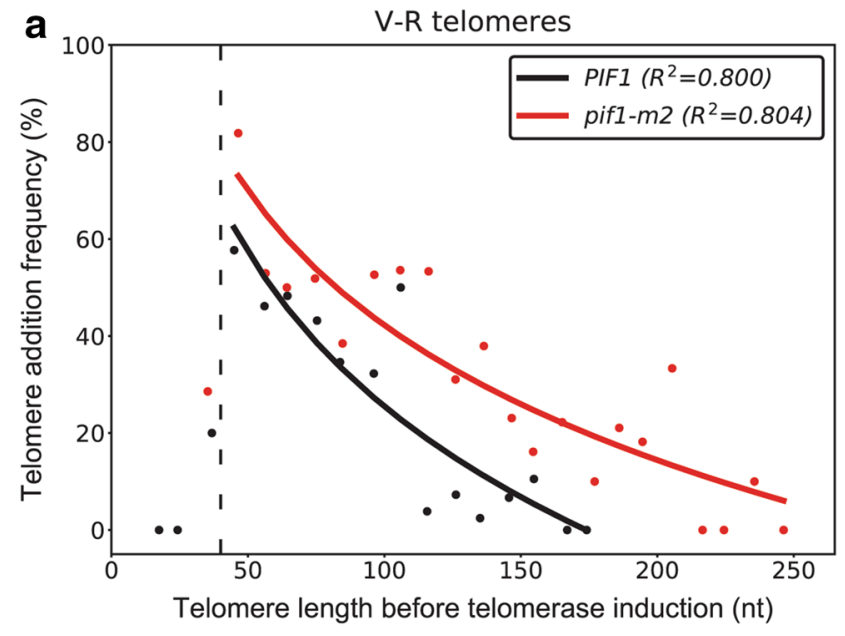

Fig. 2 Mutation of PIF1 increases the frequency of telomere extension. Telomere V-R (a) and telomere VI-R (b) sequences obtained from the iSTEX analysis of wild-type and pif1-m2 cells (Strecker et al. 2017) were binned into groups of $10 \mathrm{nt}$ in size according to telomere length before telomerase induction. Groups containing less than five telomeres were excluded from this analysis. Frequency of extension and average telomere length before telomerase induction were calculated and plotted for each group. Logarithmic

A telomere length-independent regulation of telomerase activity by Pif1 is also in conflict with the idea that Pif1 is active only below the DSB-telomere threshold to avoid telomere sequence addition at DSBs (Strecker et al. 2017). However, the genetic assay to monitor telomere addition (which measures the ability of telomerase to add a telomere to a DNA end generated by the HO endonuclease, thereby allowing the cell to grow) might be less

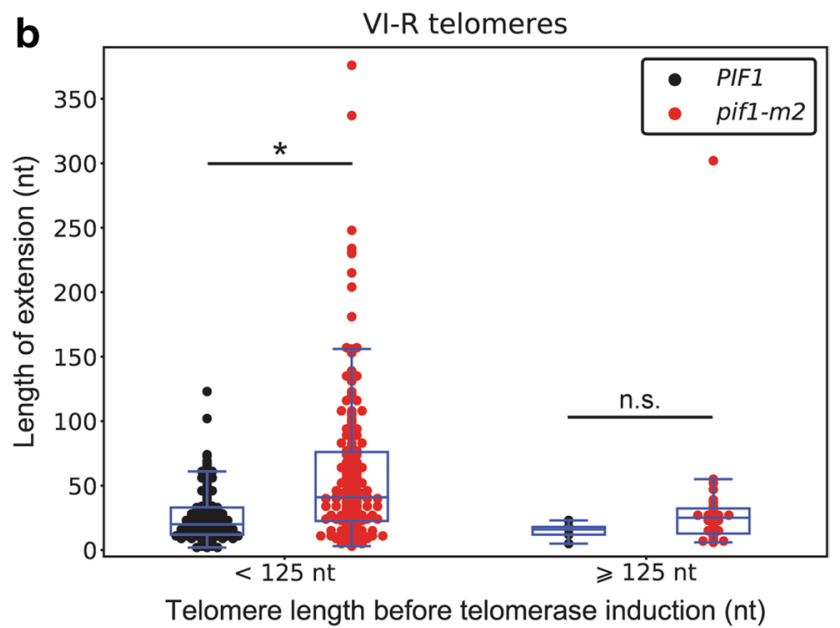

ing to telomere length prior to telomerase induction $(<125$ and $\geq 125$ nt). Statistical significance was determined using a two-tailed MannWhitney $U$ test. ${ }^{*} p$ value $<0.00001$ and $n s$ not significant

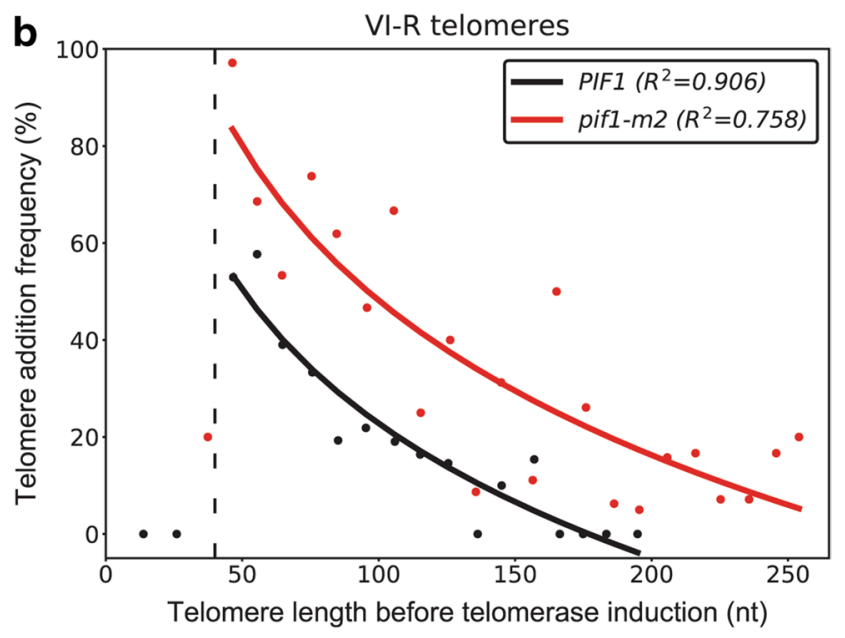

regression curves for each data set were determined using Microsoft Excel. The equations of the curves for telomere V-R are $y=-46.06 \ln (x)+237.61$ (wild-type) and $y=-40.13 \ln (x)+227.05$ (pif1-m2). The equations of the curves for telomere VI-R are $y=-39.89 \ln (x)+206.47$ (wild-type) and $y=-46 \ln (x)+259.99$ (pifl$m 2$ ). $R^{2}$ indicates coefficient of determination. Telomeres shorter than $40 \mathrm{nt}$ before telomerase induction, below the DSB-telomere threshold (dashed line), were excluded from the regression analysis

sensitive to detect Pif1 activity as compared to the iSTEX assay. While the iSTEX assay detects telomerase-mediated extension events during a 2 -h window, the inducible HO method allows a much longer time for a telomere addition event to occur: telomere addition can take place at any time during the 4-h growth period in galactose-containing medium to induce the HO cut, and within a few hours after plating, which would still give enough time for a 
cell to add a telomere to the DSB and form a colony at the end of the 3-day experiment. Therefore, telomere addition frequency in the inducible $\mathrm{HO}$ cut method is already very high at a DNA end adjacent to telomeric sequence above the DSB-telomere threshold ( $90 \%$ at a DNA end with $34 \mathrm{bp}$ of telomeric sequence; Strecker et al. 2017), making a further increase in frequency undetectable upon mutation of PIF1.

In summary, we have found that Pif 1 inhibits telomerase in a similar way across all telomere lengths, indicating that its function on telomerase inhibition does not depend on telomere length. However, several questions remain unanswered. How does phosphorylation of Pif1 only affect its activity at DNA ends below the DSB-telomere threshold? If Pif 1 is acting on both sides of this threshold, how is the threshold set? As we previously proposed (Strecker et al. 2017), a DNA end below the DSB-telomere threshold is unable to efficiently recruit or activate telomerase for a still unclear reason, and Pif1 activity ensures that telomerase is tightly inhibited. Above the threshold, telomerase recruitment/activation is strong, making Pif1 largely irrelevant. The difference above and below the threshold is independent of the interaction between Cdc13 and telomerase (Strecker et al. 2017), so, perhaps, it is recruitment of $\mathrm{Cdc} 13$ itself that is important. Furthermore, characterizing the role of Pif 1 in telomerase inhibition is complicated by the telomerase-independent functions of Pif1, which could indirectly affect telomerase activity. Most of its telomerase-independent functions-Okazaki fragment processing, DNA end resection, break-induced replication, G-quadruplex unwinding, and destabilization of R-loops-are either known to or have been proposed to occur at telomeres as well (Dewar and Lydall 2010; Geronimo and Zakian 2016). Further work will be required to characterize what role Pif1 plays in each of these processes at telomeres.

Pif1 helicases are highly conserved and its family members are identified from bacteria to humans (Bochman et al. 2010). Human Pif1, which has $24 \%$ sequence identify to Pif1 in S. cerevisiae (Bochman et al. 2010), was shown to bind to telomeric sequences in vitro (Zhang et al. 2006) and to interact with the catalytic subunit of telomerase (Mateyak and Zakian 2006). These observations, together with the fact that telomerase is upregulated in $~ 90 \%$ of human cancers (Hanahan and Weinberg 2011), make Pif1 and its associated proteins possible targets for future therapies involving the alteration of telomerase activity.

Acknowledgements We would like to thank Guillaume Gaillard for his help with the data representation. This work was supported by a European Research Council Starting grant (to KP) and a Netherlands Organisation for Scientific Research Vidi grant (to MC).
Open Access This article is distributed under the terms of the Creative Commons Attribution 4.0 International License (http://creativecommons.org/licenses/by/4.0/), which permits unrestricted use, distribution, and reproduction in any medium, provided you give appropriate credit to the original author(s) and the source, provide a link to the Creative Commons license, and indicate if changes were made.

\section{References}

Armanios M, Blackburn EH (2012) The telomere syndromes. Nat Rev Genet 13:693-704. doi:10.1038/nrg3246

Blasco MA (2005) Telomeres and human disease: ageing, cancer and beyond. Nat Rev Genet 6:611-622. doi:10.1038/nrg1656

Bochman ML, Sabouri N, Zakian VA (2010) Unwinding the functions of the Pif1 family helicases. DNA Repair, Helicase and translocases required for the maintenance of genome stability. 9:237-249. doi:10.1016/j.dnarep.2010.01.008

Boulé JB, Vega LR, Zakian VA (2005) The yeast Pif1p helicase removes telomerase from telomeric DNA. Nature 438:57-61. doi:10.1038/nature04091

Budd ME, Reis CC, Smith S, Myung K, Campbell JL (2006) Evidence suggesting that Pif1 helicase functions in DNA replication with the Dna2 helicase/nuclease and DNA polymerase $\delta$. Mol Cell Biol 26:2490-2500. doi:10.1128/ MCB.26.7.2490-2500.2006

Chang M, Arneric M, Lingner J (2007) Telomerase repeat addition processivity is increased at critically short telomeres in a Tel1dependent manner in Saccharomyces cerevisiae. Genes Dev 21:2485-2494. doi:10.1101/gad.1588807

Dewar JM, Lydall D (2010) Pif1- and Exo1-dependent nucleases coordinate checkpoint activation following telomere uncapping. EMBO J 29:4020-4034. doi:10.1038/emboj.2010.267

Ferreira MG, Miller KM, Cooper JP (2004) Indecent exposure: when telomeres become uncapped. Mol Cell 13:7-18. doi:10.1016/ S1097-2765(03)00531-8

Geronimo CL, Zakian VA (2016) Getting it done at the ends: Pif1 family DNA helicases and telomeres. DNA Repair (Amst). doi:10.1016/j.dnarep.2016.05.021

Greider CW, Blackburn EH (1985) Identification of a specific telomere terminal transferase activity in Tetrahymena extracts. Cell 43:405-413

Hanahan D, Weinberg RA (2011) Hallmarks of cancer: the next generation. Cell 144:646-674. doi:10.1016/j.cell.2011.02.013

Kim NW, Piatyszek MA, Prowse KR, Harley CB, West MD, Ho PL, Coviello GM, Wright WE, Weinrich SL, Shay JW (1994) Specific association of human telomerase activity with immortal cells and cancer. Science 266:2011-2015. doi:10.1126/science.7605428

Li JR, Yu TY, Chien IC, Lu CY, Lin JJ, Li HW (2014) Pif1 regulates telomere length by preferentially removing telomerase from long telomere ends. Nucleic Acids Res 42:8527-8536. doi:10.1093/nar/gku541

Makovets S, Blackburn EH (2009) DNA damage signalling prevents deleterious telomere addition at DNA breaks. Nat Cell Biol 11:1383-1386. doi:10.1038/ncb1985

Marcand S, Gilson E, Shore D (1997) A protein-counting mechanism for telomere length regulation in yeast. Science 275:986-990. doi:10.1126/science.275.5302.986

Mateyak MK, Zakian VA (2006) Human PIF helicase is cell cycle regulated and associates with telomerase. Cell Cycle 5:27962804. doi:10.4161/cc.5.23.3524

Myung K, Chen C, Kolodner RD (2001) Multiple pathways cooperate in the suppression of genome instability in Saccharomyces cerevisiae. Nature 411:1073-1076. doi:10.1038/35082608 
Nugent CI, Hughes TR, Lue NF, Lundblad V (1996) Cdc13p: a single-strand telomeric DNA-binding protein with a dual role in yeast telomere maintenance. Science 274:249-252

Paeschke K, Capra JA, Zakian VA (2011) DNA replication through G-Quadruplex Motifs is promoted by the Saccharomyces cerevisiae Pif1 DNA helicase. Cell 145:678-691. doi:10.1016/j. cell.2011.04.015

Phillips JA, Chan A, Paeschke K, Zakian VA (2015) The Pif1 helicase, a negative regulator of telomerase, acts preferentially at long telomeres. PLOS Genet 11:e1005186. doi:10.1371/journal. pgen.1005186

Schulz VP, Zakian VA (1994) The Saccharomyces PIF1 DNA helicase inhibits telomere elongation and de novo telomere formation. Cell 76:145-155. doi:10.1016/0092-8674(94)90179-1

Strecker J, Stinus S, Caballero Pliego, Szilard M, Chang RK, Durocher M, D (2017) A sharp Pif1-dependent threshold separates DNA double-strand breaks from critically short telomeres. eLife 6:e23783. doi:10.7554/eLife.23783

Teixeira MT, Arneric M, Sperisen P, Lingner J (2004) Telomere length homeostasis is achieved via a switch between telomerase-extendible and -nonextendible states. Cell 117:323335. doi:10.1016/S0092-8674(04)00334-4

Wellinger RJ, Zakian VA (2012) Everything you ever wanted to know about Saccharomyces cerevisiae telomeres: beginning to end. Genetics 191:1073-1105. doi:10.1534/genetics.111.137851

Wotton D, Shore D (1997) A novel Rap1p-interacting factor, Rif2p, cooperates with Rif1p to regulate telomere length in Saccharomyces cerevisiae. Genes Dev 11:748-760. doi:10.1101/gad.11.6.748

Zhang W, Durocher D (2010) De novo telomere formation is suppressed by the Mec1-dependent inhibition of $\mathrm{Cdc} 13$ accumulation at DNA breaks. Genes Dev 24:502-515. doi:10.1101/gad.1869110

Zhang D-H, Zhou B, Huang Y, Xu L-X, Zhou J-Q (2006) The human Pif1 helicase, a potential Escherichia coli RecD homologue, inhibits telomerase activity. Nucleic Acids Res 34:1393-1404. doi:10.1093/nar/gkl029

Zhou JQ, Monson EK, Teng SC, Schulz VP, Zakian VA (2000) Pif1p helicase, a catalytic inhibitor of telomerase in yeast. Science 289:771-774. doi:10.1126/science.289.5480.771 